Supporting Information

\title{
Molecular Sensitivity of Near-Field Vibrational Infrared Imaging
}

Chih-Feng Wang ${ }^{\ddagger}$, Bijesh Kafle ${ }^{\ddagger \dagger}$, Tefera E. Tesema ${ }^{\ddagger \dagger}$, Hamed Kookhaee ${ }^{\ddagger \dagger} \dagger$ and Terefe

$$
\text { G. Habteyes }{ }^{\ddagger \dagger \dagger *}
$$

†Department of Chemistry and Chemical Biology, and ${ }^{\ddagger}$ Center for High Technology

Materials, University of New Mexico, Albuquerque, New Mexico 87131, Unites States

*Corresponding email: habteyes@unm.edu

\section{Content}

Figure S1 AFM topography and mechanical phase along with near-field amplitude and phase images at $\mathrm{m}=2-4$ of $\mathrm{P} 4 \mathrm{VP}$ on gold and silicon substrates

Figure S2 Near-field signal as a function of tip-substrate separation in P4VP or air medium.

Figure S3 Comparison of phase contrast of P4VP on gold and silicon.

Figure S4 Illustration that more chemical details can be resolved at higher order of demodulation.

Figure S5 Near-field images and line profiles of ultrathin P4VP film on gold

Figure S6 Contrasts of nanoscale features within the P4VP film.

Figure S7 Line profiles showing the variation in the optical contrast due to nanoscale features within the P4VP film. 
(a) Au-P4VP
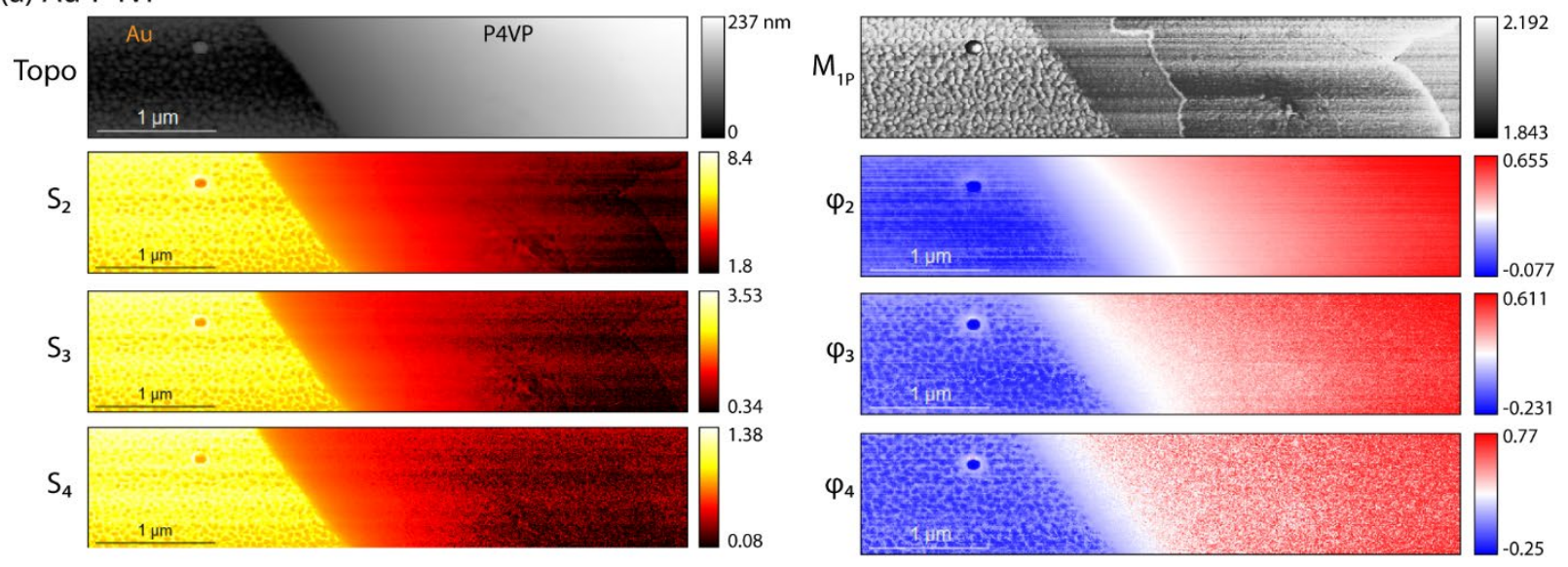

(b) Si-P4VP
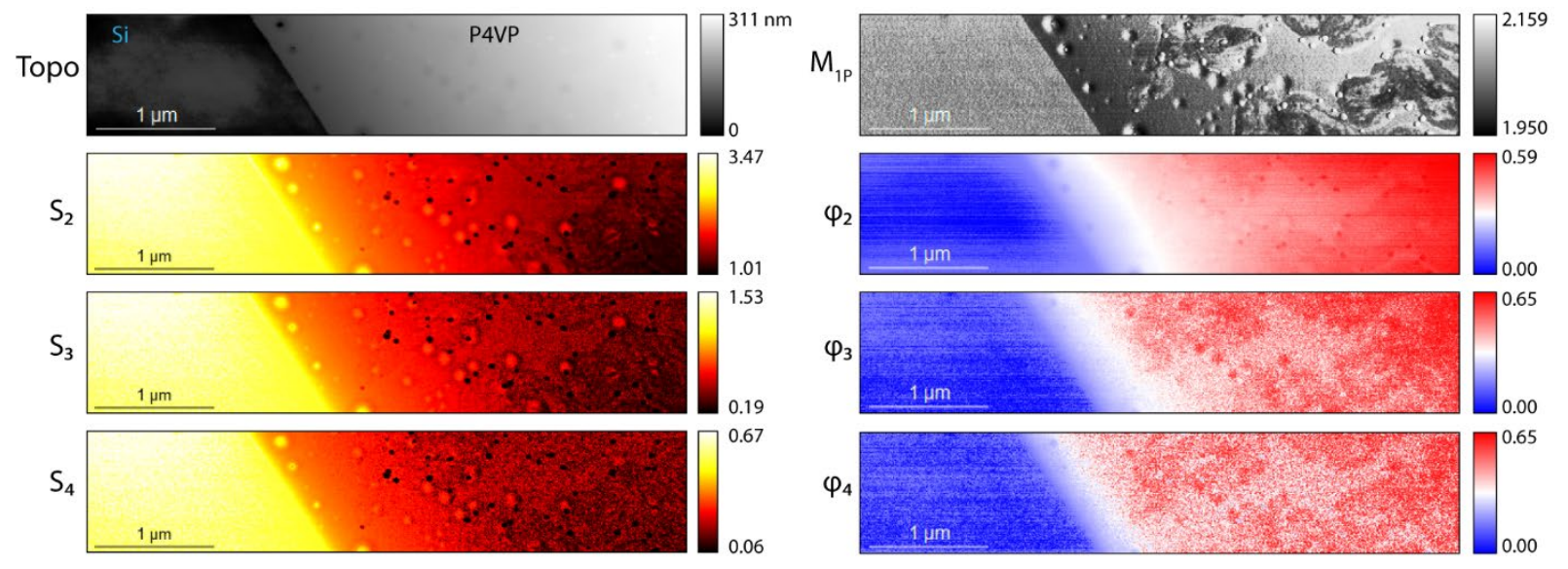

Figure S1 AFM topography (topo), mechanical phase $\left(\mathrm{M}_{1 \mathrm{P}}\right)$, near-field amplitude $\left(\mathrm{S}_{\mathrm{m}}\right)$ and nearfield phase $\left(\varphi_{\mathrm{m}}\right)$ on (a) gold, and (b) silicon substrates. Note that more details of chemical contrast are revealed in the near-field phase images at higher order of demodulation $\mathrm{m}$. 

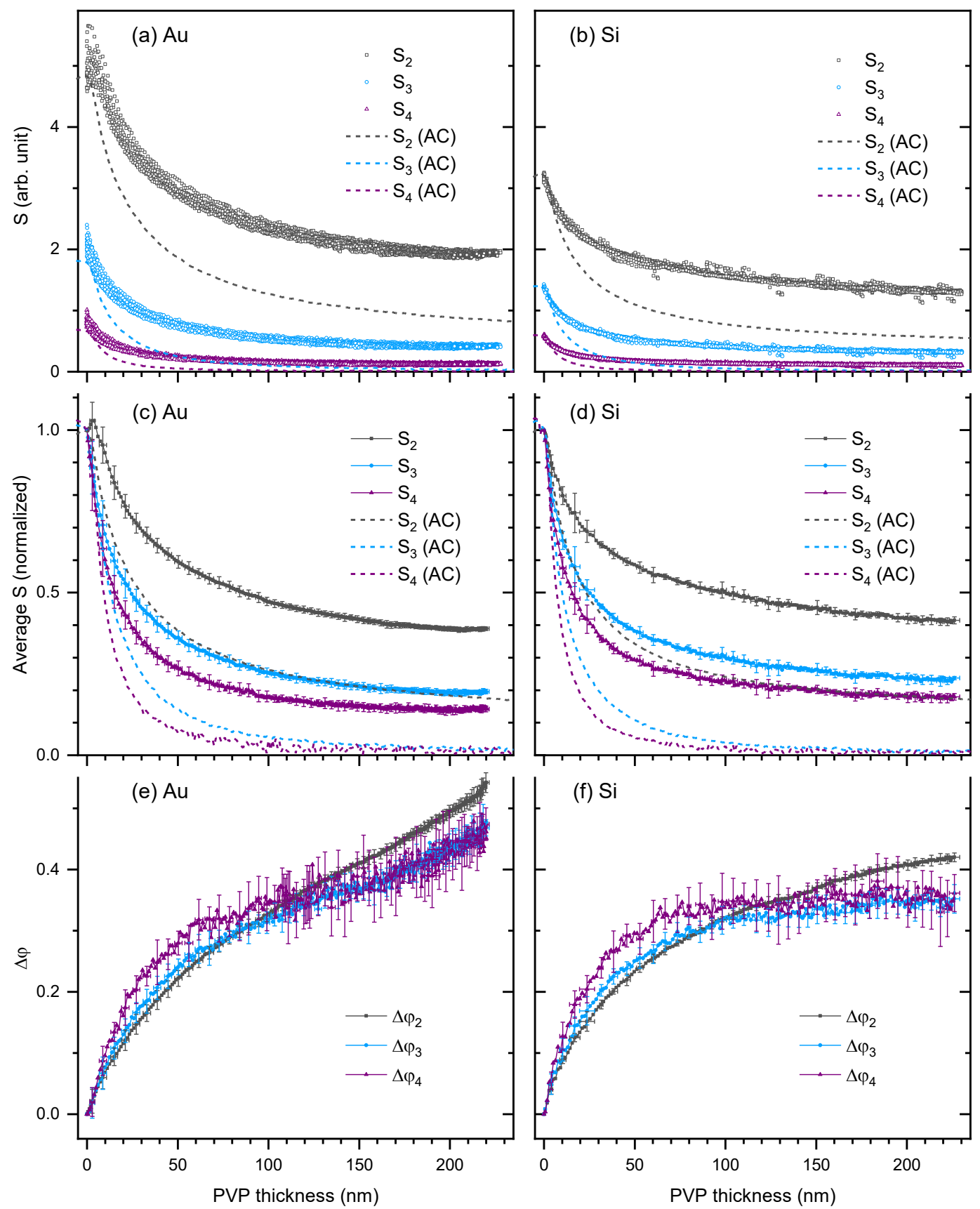

Figure S2 Dependence of near-field amplitude and phase on P4VP thickness. (a, b) Data points of ten horizontal lines across the amplitude images in Figure S1a and S1b. The dashed lines are approach curves (AC) showing the amplitude decay as the tip-substrate separation is increased in air. (c, d) Average of the data points in (a, b) after normalizing by their maximum (the corresponding amplitudes on the substrates). (e, f) Average data points of the phase contrasts that correspond to the same lines as the amplitudes in $(\mathrm{a}, \mathrm{b})$. 

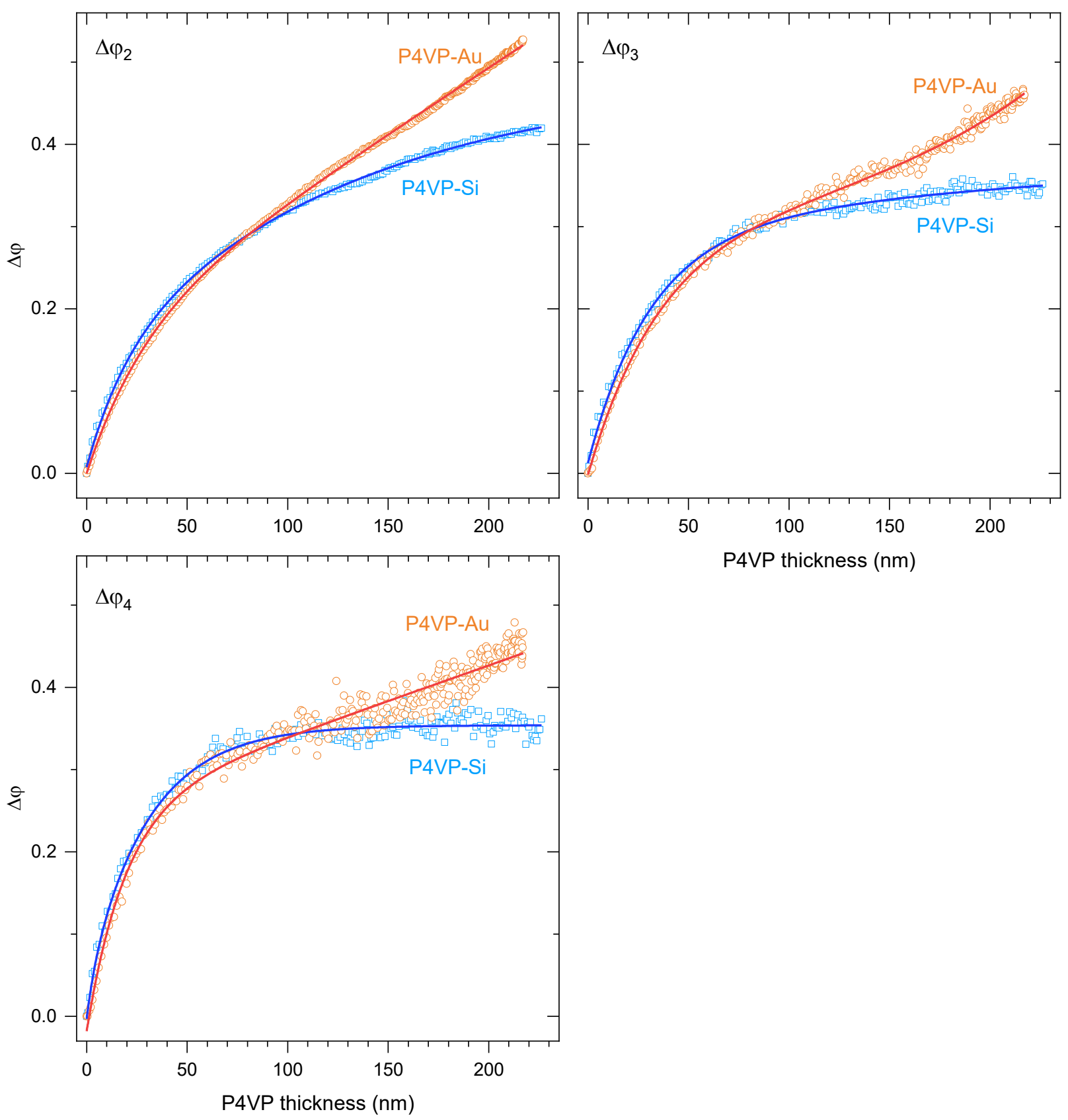

Figure S3 Comparison of near-field phase changes due to P4VP on gold and silicon at different harmonics. The solid lines in each case are bi-exponential function fit to the corresponding data. 


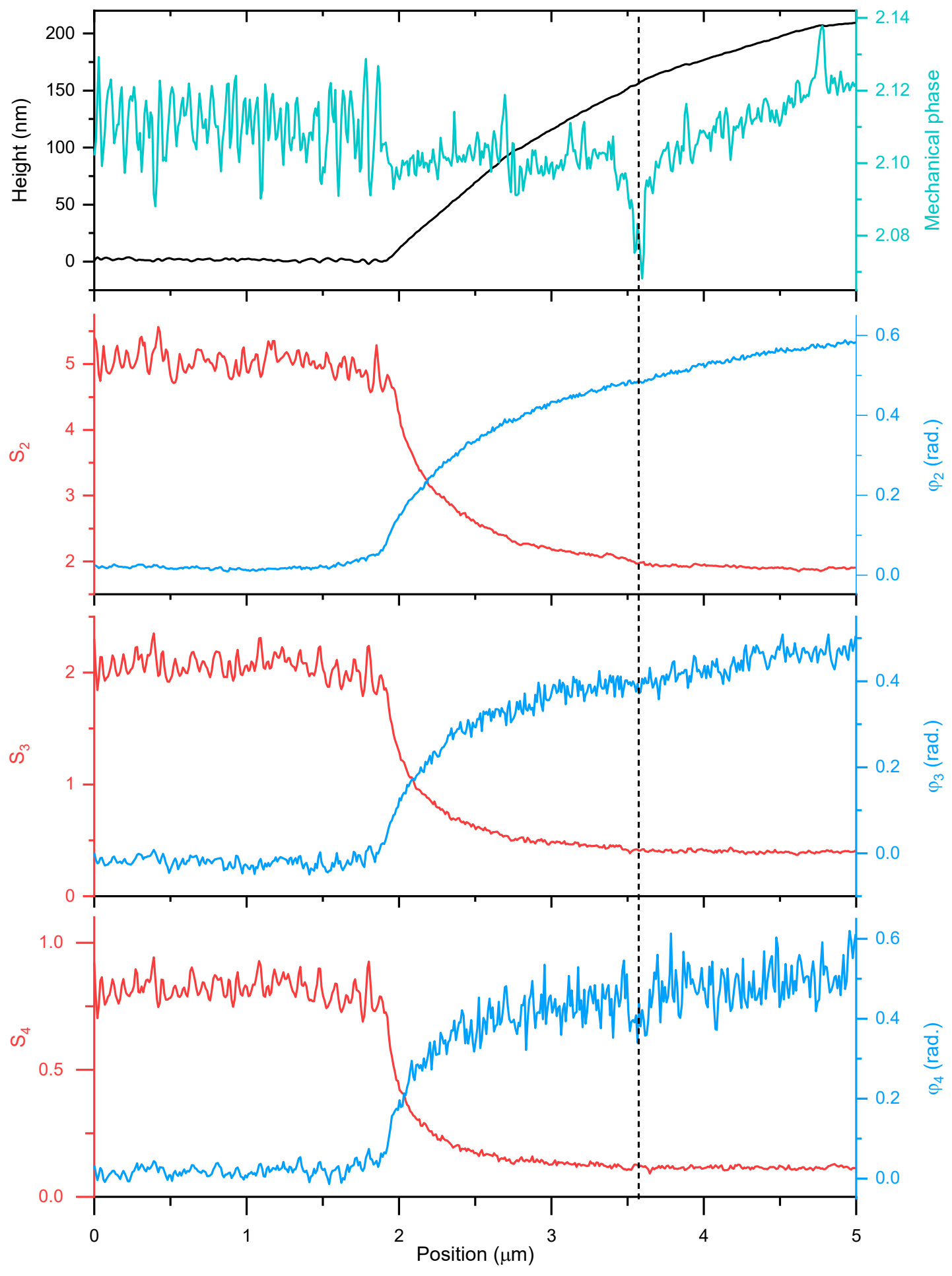

Figure S4 Line profiles showing that more details of chemical contrast are revealed with increasing order of signal demodulation as the background suppression becomes increasingly effective. The vertical dashed line indicates a point where the dip observed in the mechanical phase is resolved at $\mathrm{m}=4$ but not at $\mathrm{m}=2$ and 3 . 

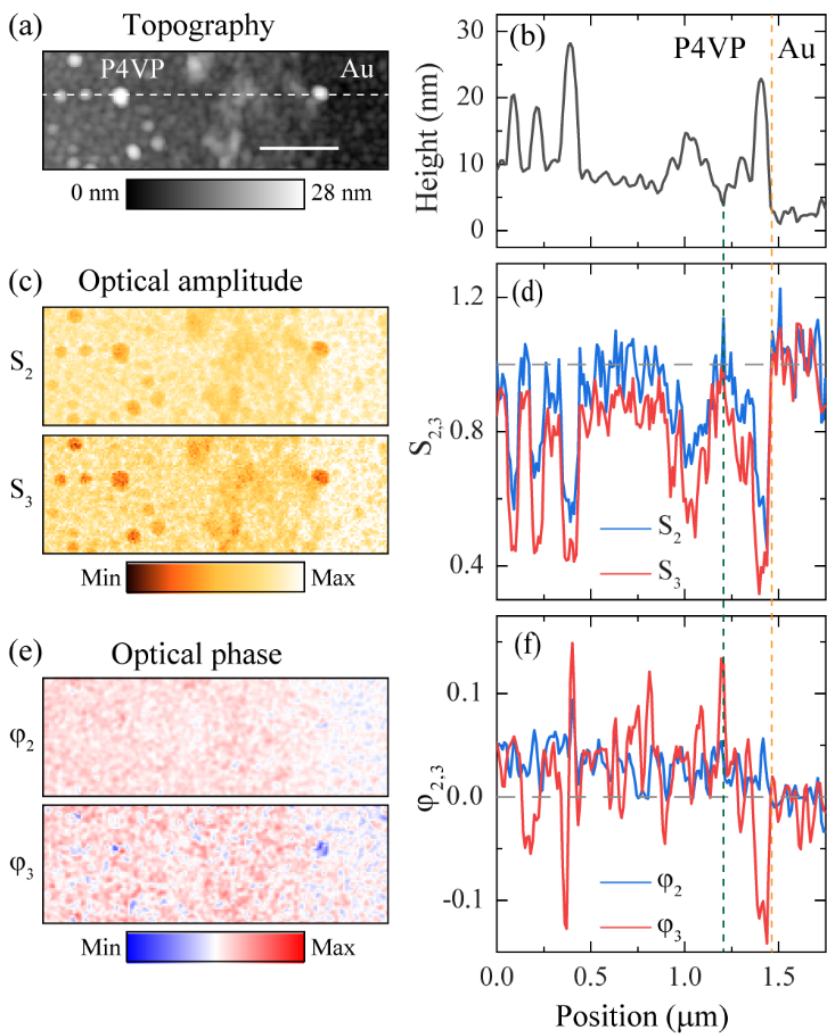

Figure S5 The images are same as in Figure 5 in the main manuscript. Line profiles in (b) - (f) show that the phase contrast does not necessarily correlate with the topographic height. For example, the lowest point that has about $3 \mathrm{~nm}$ P4VP thickness gives one of the highest points in the phase contrast as marked by the vertical green dashed line. We note that the film thickness is comparable to the roughness of the gold surface. 

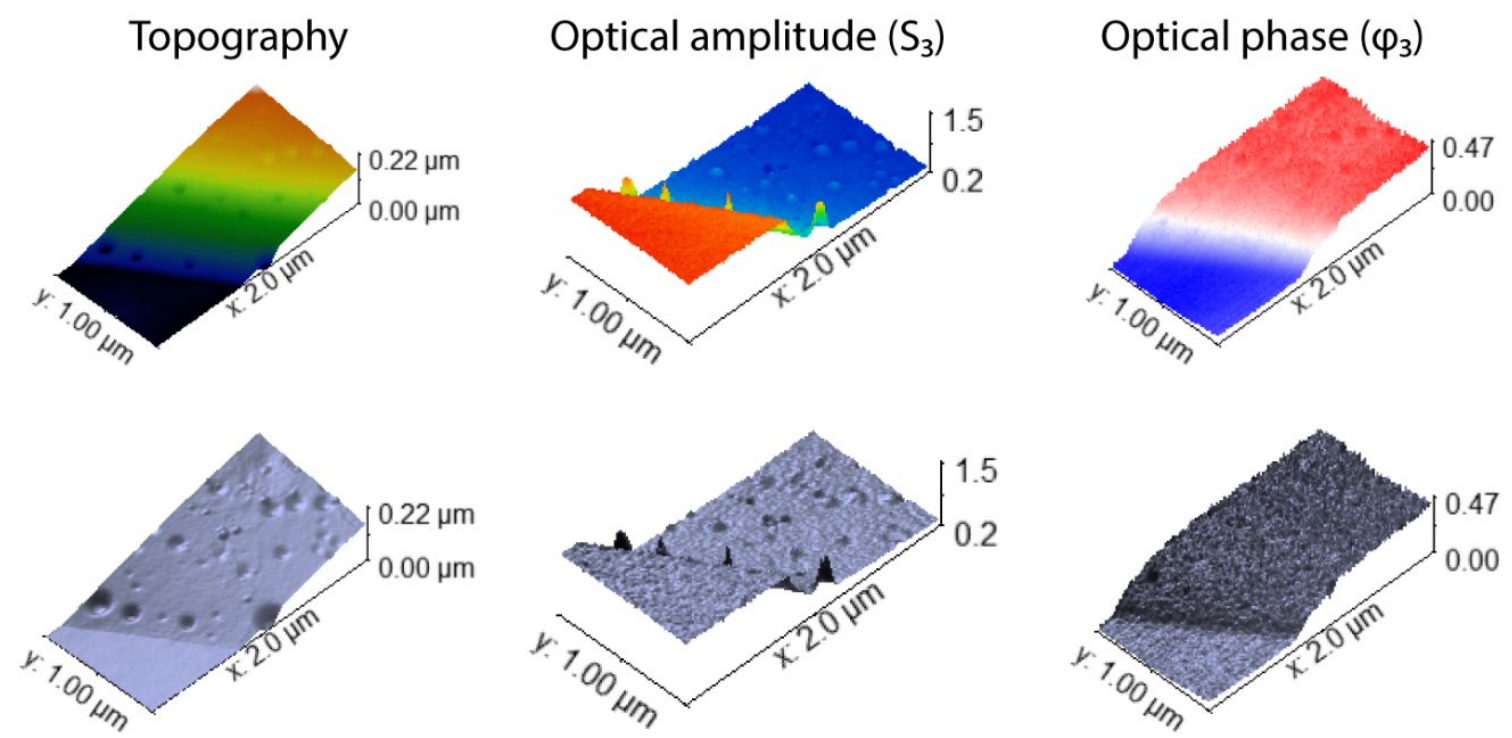

Figure S6 Contrasts of nanoscale features within the P4VP film. Top panels: 3-dimensional plots in which the variation in height, optical amplitude and optical phase is represented with color gradient. Bottom panels: same as top panels applying lighting effect (using Gwyddion software) to enhance the visibility of the nanoscale dips and peaks within the polymer film. See details of the contrasts in the representative line profiles in Figure S7. 


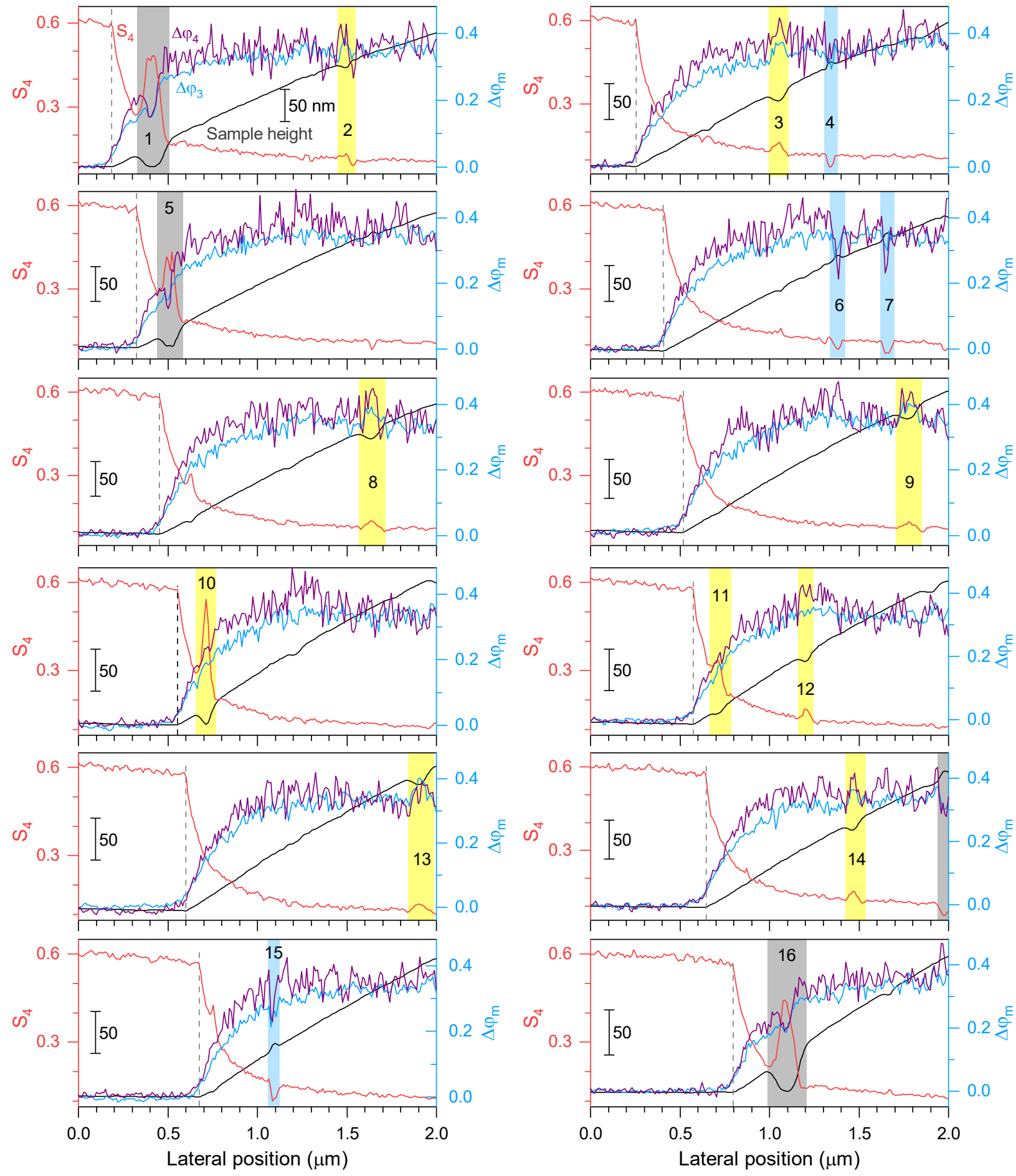

Figure S7 Line profiles across different rows in the images in Figure S6. In all plots, black lines represent the topography for which the height scale is shown by the $50 \mathrm{~nm}$ scale bar. The red lines indicate the optical amplitude, and the blue and purple lines the $3^{\text {rd }}$ and $4^{\text {th }}$ order phase contrast. For illustrating the IR contrasts due to nanoscale features within the bulk film, sixteen dips or peaks in the topography are shaded. The gray shades (1,5 and 16) indicate expected trend; that is a decrease in sample height leads to an increase in the optical amplitude and a decrease in the phase 
contrast according to the general trend discussed in Figures 2 and 3. The yellow and cyan shades indicate anomaly in the phase contrast. In the yellow shades $(2,3,8,9,10,11,12,13$ and 14), the dips in the topography correspond to peaks in both optical amplitude and phase. In the cyan shades $(4,6,7$ and 15), high points in the topography leads to low points in both amplitude and phase contrasts. 